\title{
LA CIENCIA FICCIÓN
}

\section{Y LA ENSEÑANZA DE LAS CIENCIAS}

\section{SCIENCE FICTION AND THE TEACHING OF SCIENCE}

\author{
Ma Francisca Petit Pérez \\ mfranpe@alumni.uv.es \\ Jordi Solbes Matarredona \\ Departament de didàctica de les Ciencies Experimentals i Socials. \\ Universitat de València \\ jordi.solbes@uv.es
}

RESUMEN: Este trabajo constituye una primera aproximación a la ciencia en la ciencia ficción desde el punto de vista del alumnado y qué posibilidades ofrece como recurso didáctico. Sondeamos, por tanto, si los alumnos conocen y han visto cine de ciencia ficción, así como si este factor influye en sus actitudes hacia la ciencia y los científicos y su opinión sobre las consecuencias de los avances científicos en el futuro. Asimismo, nos planteamos si los estudiantes perciben las visiones deformadas de la ciencia y los científicos que transmite el cine de ciencia ficción. Pretendemos, asimismo, estudiar si se utiliza la ciencia ficción como recurso didáctico.

PALABRAS CLAVE: Comprensión pública de la ciencia, ciencia ficción, actitudes hacia la ciencia.

SUMMARY: This work consists of a first approach to Science in science-fiction from the students' point of view and researches on which possibilities it offers as a teaching aid. Therefore, we have polled whether the students know about and have seen science fiction cinema, as well as whether this factor has an influence on their attitudes toward Science and scientists as well as their opinion about the consequences of scientific progress in the future. In addition, we wonder whether students are aware of the twisted views of Science and scientists that science-fiction cinema transmits. We also consider whether science-fiction is used like a teaching aid.

KEY WORDS: Public understanding of Science, science fiction, attitudes toward Science. 


\section{INTRODUCCIÓN}

En el Informe Rocard y otros (2007) se alerta sobre el «peligro capital para el futuro de Europa» que supone la disminución de jóvenes que estudian ciencias y se afirma que los «orígenes de esta situación pueden encontrarse en la manera como se enseña la ciencia». Y esta manera es la «enseñanza magistral» que ha impregnado profundamente a los profesores a lo largo de los muchos años en que la han seguido como alumnos. Se trata de una formación ambiental que ha tenido un gran peso por su carácter reiterado y no estar sometida a una crítica explícita, apareciendo por ello como «natural», sin que llegue a ser cuestionada efectivamente (Furió y Carnicer, 2002; Furió et al., 2008; Hewson y Hewson, 1987; Porlán et al., 1998). Sin embargo, cada vez son más los educadores que intentan salir de ese círculo de desinterés que se genera en el aula si la materia y el método de impartirla no motivan ni a los alumnos ni a los propios profesores (Banet, 2007).

Pero como señalan Solbes y otros (2007), este desinterés y abandono es un fenómeno complejo, multicausal en el que influye no sólo la manera en que se enseñan y aprenden las ciencias, sino también la imagen pública de la ciencia, la poca importancia dada por las autoridades educativas a la ciencia en el sistema educativo, las relaciones entre el sexo del alumnado y su elección de estudios de ciencias, etc.

Uno de los grandes problemas que encontramos en el campo de la didáctica de las ciencias ha sido y sigue siendo que la sociedad en general, y los alumnos en particular, tienen una imagen muy negativa de la ciencia a la que consideran difícil, aburrida, sólo apta para genios y, por otra parte, la consideran la mayor responsable de algunos de los grandes problemas de nuestro tiempo, como el armamentismo, la contaminación, etc. (Solbes y Traver, 2001; Solbes et al, 2007). Vamos a analizar en qué medida contribuyen a esta imagen los libros, cine, series de TV, cómics, etc. de ciencia ficción (CF).

Por todo ello, nos planteamos los siguientes problemas:

- ¿Qué entienden los y las estudiantes por ciencia ficción? Y ¿qué ciencia ficción conocen?

- ¿Qué imagen de la ciencia y de los científicos y qué visión sobre el futuro captan los alumnos a través de la CF?

- ¿Qué opinan los profesores sobre la utilización de la ciencia ficción como recurso didáctico? Y, por último, ¿se utiliza la ciencia ficción en los libros de texto?

\section{MARCO TEÓRICO}

La fundamentación teórica de este trabajo se refiere, en primer lugar, a la cuestión de las actitudes.

La distinción entre actitudes científicas y actitudes hacia las ciencias ha sido ampliamente abordada por la investigación educativa (Furió y Vilches, 1997; Pozo y Gómez, 1998; Simpson et al., 1994; Solbes, 1999). También se ha investigado el hecho de que las actitudes hacia las ciencias sean negativas (Yager y Penick, 1986; Solbes y Vilches, 1997; Fensham, 2004; Sjøberg y Schreiner, 2006) y que, en consecuencia, el abandono de los estudios de ciencias no ha hecho más que aumentar (Matthews, 1990; Dunbar, 1999; Solbes, Monserrat y Furió, 2007; Vázquez y Manassero, 2008).

La mayoría de estos trabajos atribuyen las actitudes negativas y el desinterés básicamente a los contenidos y la metodología de la enseñanza. Pero ya hemos señalado que en ese desinterés influyen otros factores, como:

- una imagen pública bastante negativa de la ciencia, a la que se atribuyen problemas de nuestro tiempo como los armamentos, la contaminación, etc.,

- un bajo estatus de la ciencia en el sistema educativo español,

- una sensible reducción de las chicas en los estudios científico-técnicos (no en los de ciencias de la naturaleza y la salud). 
Nos centraremos en el primero de ellos y trataremos de averiguar cómo influye el género de CF, muy presente en importantes medios de comunicación (cine, TV...) en la comprensión pública de la ciencia y los científicos por parte de los estudiantes de secundaria.

La segunda línea de fundamentación conecta con algunos trabajos (Perales y Vílchez, 2005; Pro y Ezquerra, 2005) sobre el uso de recursos audiovisuales para mejorar la enseñanza y aprendizaje de las ciencias y, más en concreto, con los que se centran en la utilización de la CF y su contribución a la motivación del alumnado. En particular, en nuestro país hay algunos artículos que valoran o utilizan la CF como recurso didáctico, pero apenas se ha investigado si los estudiantes conocen la CF, si les interesa y cómo contribuye la CF a cuestiones CTS como la imagen de la ciencia y de los científicos.

Los ejemplos más abundantes se dan en la enseñanza superior mediante el estudio guiado de un relato. Por ejemplo, en el ámbito de la relatividad especial, que se presta con facilidad para su uso por la ingente cantidad de materiales y situaciones noveladas susceptibles de análisis: viajes espaciales, paradojas del estilo de la de los gemelos, comunicaciones, aspecto de cuerpos en movimiento, adición de velocidades, etc. (Nicholls, 1987). La experiencia llevada a cabo por Hellstrand y Ott (1995) consistió precisamente en detectar diferencias significativas en el aprendizaje de aspectos cualitativos del tema tras la simple lectura de un relato. Valoraron muy positivamente el sustancial incremento del interés de los alumnos en la formalización cuantitativa de los conceptos, con respecto a quienes no participaron en la experiencia. En España diversos profesores universitarios de Física, Informática, etc. (Barceló, 2000 y 2005; Moreno y José, 2002) han hecho uso de relatos en la línea de mejorar el aprendizaje de conceptos, detectar ideas alternativas, etc. En esta misma línea se ha utilizado como recurso didáctico en Educación Secundaria Obligatoria (Bacas y otros, 1993 y 1997).

Es más reciente el uso de los cómics de superhéroes (Kakalios, 2006) o del cine o las series de CF tanto a nivel universitario (Palacios, 2007) y secundaria (García Borras, 2006), para facilitar el aprendizaje de conceptos, etc. La mayoría de estos autores insisten en el carácter motivador del recurso y en que no está suficientemente explotado pero que constituye una buena herramienta como recurso didáctico alternativo a las clases «convencionales» de las asignaturas de ciencias (García Borras, 2006; Sierra, 2007).

Sin embargo, la opinión que se forma el alumnado sobre los científicos, la ciencia y la responsabilidad que ésta pueda tener en cómo será el futuro, basada en la CF, no ha sido tan investigada. Por ello, nos interesa saber qué CF «consumen» los alumnos en libros, cine y televisión, siendo conscientes de que estos últimos son los medios de entretenimiento más utilizados, lo que se confirma con las encuestas realizadas por el Ministerio de Cultura sobre los hábitos culturales de los españoles.

Pero para poder clasificar las respuestas del alumnado y el profesorado sobre la CF que conocen es necesario resolver previamente la siguiente cuestión: ¿Qué se entiende por ciencia ficción y qué cine se puede clasificar como ciencia ficción?, ya que, debido a la subjetividad que implica la creación literaria (y cinematográfica), encontramos muchas definiciones para la literatura de CF. Todas las podríamos referir también al cine de CF. Alguna de estas definiciones se recogen en la Enciclopedia de Ciencia Ficción de John Clute y Peter Nicholls (1995), de las que destacamos la definición dada por Asimov: "la ciencia ficción es la rama de la literatura que trata de la respuesta humana a los cambios en el nivel de la ciencia y la tecnología». Queremos destacar de todas maneras que prácticamente todas las definiciones tienen dos puntos, explícitos o no, en común que son el futuro y los descubrimientos o invenciones más o menos fundamentadas científicamente.

Si pretendemos clasificar la CF nos topamos directamente con el problema de las discrepancias sobre libros o películas incluidas en el género. Basándonos en autores con una clara orientación científica (Barceló, 2000; José, 2006; Moreno, 2006), proponemos las siguientes categorías en que encuadrar las películas, novelas y series de ciencia ficción. Son las siguientes: 
1. Viajes (o conquista) espacial. (Posibles: 2001, Misión a Marte, Planeta Rojo; ficción: Star Trek; Star Gate, Babylon 5).

2. Extraterrestres (Ultimátum a la Tierra, Encuentros en la 3. a fase, ET, Contact, Alien; invasiones: Mars attack, Independence day, La guerra de los mundos).

3. Futuro (1984, Fahrenheit, Mundo feliz, Planeta de los simios).

4. Robots y androides (Metrópolis, Planeta prohibido, Blade Runner, El hombre Bicentenario, Yo robot, IA, Terminator).

5. Viajes en el tiempo (El tiempo en sus manos, Regreso al futuro, Déjà vú).

6. Computadores (Colossus, Tron, Juegos de Guerra, Matrix).

7. Viajes al interior del cuerpo humano (Viaje alucinante).

8. Experimentación con humanos (Frankenstein, Dr. Jeckyll, La isla del Dr. Moreau, Mundo feliz; La mosca, que también se incluyen en el género de cine de terror).

9. Ingeniería genética, clones (Mundo feliz, Gattaca, Parque jurásico, La isla).

10. Catástrofes, desastres ecológicos (El día después, Armaggedon; geológicas: Volcano, Terremoto, Tornado).

11. Superhéroes (La masa, X-men, Los 4 fantásticos).

Las dos últimas categorías son más discutidas, en especial, las catástrofes ecológicas. En cuanto a obras de superhéroes, sí las incluimos porque muchos de ellos se originan a partir de procesos o accidentes de la ciencia y porque los científicos están muy presentes en las mismas. También es difícil encuadrar algunos títulos como Star wars, Dune, etc. que se pueden considerar espaciales, de extraterrestres, futuro y robots. Por eso algunos las denominan «space operas».

Esta clasificación no pretende incluir todas las películas de CF, pero sí las que hemos considerado más populares y las que debido a su difusión, mayor número de alumnos puede conocer, que al fin es lo que necesitamos para poder utilizar el cine de CF como recurso didáctico.

Esto excluye las obras del género fantástico, basadas principalmente en la magia, que es claramente anticientífica. Con respecto a las obras de terror, tampoco las incluimos en el género de ciencia ficción, porque muchas se basan en los llamados fenómenos paranormales; sin embargo, sí incluiremos películas basadas en experimentos científicos malogrados (categoría 8).

Por el contrario, en la Encuesta de Hábitos y Prácticas Culturales del Ministerio de Cultura, encontramos una identificación entre fantasía, en su versión mágica, y CF. Pensamos que esto lleva a una confusión que, lejos de beneficiar la dimensión científica, favorece el auge de la magia en nuestra sociedad, claramente evidenciado en el éxito de la saga literaria y cinematográfica de «Harry Potter» de Joanne K. Rowling y su gran cohorte de imitadores (Elias, 2008). Las personas, ante la complejidad de los aparatos que proporciona la tecnología y, especialmente, las TIC, y desconociendo las ideas básicas de las ciencias que los han hecho posibles, desarrollan ante ellos planteamientos de «caja negra» (Solbes et al., 2004). Es decir, apretando un botón conseguimos satisfacer fácilmente algunos deseos: iluminación, sonidos, imágenes, etc. Y éste era el principal objetivo de la magia: satisfacer necesidades sin esfuerzo, mágicamente. Por ello no deja de ser significativo que en los libros leídos por los jóvenes hace algunos años (Jules Verne, Conan Doyle) los problemas se resolvían por métodos científicos y tecnológicos. Ahora, por el contrario, en Rowling, se resuelven a golpe de varita mágica y con un montón de hechizos, contraviniendo además todas las leyes naturales (Petit, 2008). Por otra parte, muchos fenómenos científicos sin su correspondiente explicación se convierten en «trucos mágicos», y como tales son aprovechados por los profesionales del gremio. 


\section{METODOLOGÍA DE INVESTIGACIÓN}

A continuación pasamos a describir los diseños elaborados para el estudio, consistentes en un cuestionario para alumnos, entrevistas a los mismos, un cuestionario para profesores y un análisis de libros de texto.

Los cuestionarios con las técnicas usuales en la investigación educativa: elaboración de un primer borrador, examinado por expertos; realización de un ensayo piloto, para analizar la validez del cuestionario revisado y reelaboración del mismo cuestionario a la luz del resultado piloto.

Así pues, se propuso un cuestionario a 173 alumnos de cuatro centros diferentes, seleccionados de forma que uno era rural y tres urbanos, dos públicos y dos concertados, lo que responde aproximadamente a la proporción de dichos centros en el País Valenciano. Los alumnos cursaban $3 .^{\circ}$ y $4 .^{\circ}$ de ESO y $1 .^{\circ}$ y $2 .^{\circ}$ de bachillerato, de las modalidades tanto de ciencias sociales y humanísticas como de ciencias naturales y de la salud y científico-tecnológico. De estos alumnos se conoce el curso al que pertenecen, la especialidad cursada (salvo en los alumnos de 3. ${ }^{\circ}$ de ESO, que todavía no cursan especialidad) y el sexo.

Los cuestionarios se pasaron en una sesión ordinaria de clase.

Este cuestionario pretende analizar qué CF conocen los alumnos y en qué formato, así como la visión de la ciencia, los científicos y la influencia de la ciencia en el futuro que se forman a través de la CF. Los ítems se muestran en la presentación de resultados.

Se realizaron, además, entrevistas a 11 alumnos que habían contestado previamente el cuestionario para aclarar y profundizar en algunos de los resultados obtenidos en el mismo. Estos alumnos fueron elegidos al azar y las entrevistas se llevaron a cabo varios meses después de haber pasado el cuestionario, con un guión basado en las mismas preguntas de éste, a las que el alumno contestaba de forma abierta, pidiéndosele que clarificase explicaciones dudosas.

Por otro lado, se diseño y aplicó un cuestionario a 21 profesores mayoritariamente de física y química en activo, asistentes a un curso de un Centro de Profesores y 35 en formación (que cursaban el CAP de dicha especialidad) para determinar la CF que conocen, si consideran que la utilización de la CF puede ser un buen recurso didáctico y qué actividades harían utilizando la CF. Los ítems también aparecen en la presentación de resultados.

Por último, se analiza la presencia o no de ciencia ficción en 31 textos de ciencias y tecnología de secundaria obligatoria (18 libros) y de bachillerato (13 libros), de las especialidades de física y química (19 libros), biología y geología (7) y tecnología (5 libros), junto con los libros de profesor, CD-ROM y libros de actividades. Son textos de 7 de las principales editoriales (SM, Edebé, Edelvives, Bruño, Oxford, Editex, Anaya) publicados entre 2000 y 2008. El objetivo del análisis es determinar si existen actividades, comentarios, problemas, fotografías, etc., basadas en películas de CF, que los profesores puedan utilizar.

El análisis de la información obtenida se ha realizado según el tipo de requerimiento: por un lado, los cuestionarios contienen preguntas en las que hay que dar referencia de películas, libros o programas y, por otro lado, hay cuestiones de opinión. En el primer caso el análisis ha consistido en un mero recuento de los datos; para el segundo tipo de cuestiones, se consideró necesario clasificarlas en generales. Para ello, el análisis de las contestaciones a cada pregunta ha sido realizado independientemente por los dos investigadores para consensuar la adscripción de las respuestas a las diferentes categorías que se exponen en el apartado de resultados. 


\section{PRESENTACIÓN Y DISCUSIÓN DE RESULTADOS}

\section{Cuestionario de estudiantes}

El cuestionario consta de 8 preguntas. Las 4 primeras se centran en la CF que conoce el alumnado y las siguientes en visiones de la ciencia, los científicos y la influencia de la ciencia en el futuro e interés por la CF. Veamos, en primer lugar:

\section{LA CF QUE CONOCE EL ALUMNADO}

\section{1. ¿Has visto películas de ciencia ficción? ¿Cuáles?}

Después de analizar los cuestionarios y seleccionar las referencias de CF, frente a las de fantasía, aventuras y algunas inclasificables, obtenemos un total de 578 referencias específicas de CF, lo que da 3,3 referencias por cuestionario.

Destacan por el número de referencias la Guerra de las galaxias, con 90, Matrix (60), X-men (41), Yo robot (36), Spiderman (32), El día de mañana (24)...

Como ya hemos señalado, hay 78 referencias que confunden la CF con la magia y el cine de acción y aventura, ya que son muy mencionadas películas como Harry Potter, El señor de los Anillos, con 59 y 50 referencias respectivamente, Las Crónicas de Narnia, La historia interminable o Misión imposible, James Bond, El código da Vinci.

Algunas películas clásicas del género apenas han obtenido referencias. Así 2001, odisea en el Espacio (2 referencias), El planeta de los simios (9), y Blade Runner (0). No se hace referencia alguna a películas de años anteriores a los 80, a no ser que existan versiones posteriores como en el caso de La Guerra de los Mundos o El planeta de los simios.

En las entrevistas realizadas, se volvió a preguntar sobre películas de CF y 2 de los alumnos entrevistados nombran Harry Potter como CF y otro nombra Misión Imposible.

\section{2. ¿Has leido novelas de ciencia ficción? ¿Cuáles?}

El número total de referencias aportadas es de 215, pertenecientes a 52 títulos diferentes. Sin embargo, se han encontrado 46 cuestionarios con la respuesta de no sé o en blanco (NS/NC). Si contabilizamos únicamente las que se refieren a CF (105 referencias correspondientes a 30 títulos de CF), el resultado que obtenemos es de 0,6 referencias por cuestionario.

Lo primero que constatamos al comparar los resultados de esta pregunta con los de la pregunta anterior es que tanto el número de referencias como las obras referidas es mucho menor, lo que pone de manifiesto que los alumnos consumen más cine que lectura.

Destaca la novela de Laura García Gallego, Las Hijas de Tara (50 referencias) y Un mundo feliz de Aldous Huxley (34) que han sido lectura obligada en la clase de literatura en alguno de los centros donde se ha pasado el cuestionario.

Podemos constatar una mayor confusión entre géneros CF y fantasía, viendo que 110 referencias (el $51 \%$ ) corresponden a novelas de tipo mágico o fantástico, destacando la serie de Harry Potter con 30 referencias. 


\section{3. ¿Conoces alguna serie de ciencia ficción que se haya emitido en TV? ¿Cuál?}

El número total de referencias aportadas es de 322, repartidas entre 59 títulos diferentes (20 de CF) en los 173 cuestionarios analizados, con un total de 20 cuestionarios NS/NC. En este apartado los alumnos han incluido referencias a series de dibujos animados y anime japonés (68 referencias), series con ingredientes científicos, pero no futuristas (CSI, House) (20) y nuevamente series de fantasía y magia (50), como Embrujadas (25 ref.), Sabrina (13) y Ala dina (2). Contabilizando sólo las respuestas que consideramos se refieren a series de CF (172 respuestas), obtenemos 1 respuesta por cuestionario. Las más mencionadas son Smallville (54) de superhéroes, la clásica Star Trek (25), Star Gate (18).

\section{Indica otras series o programas de contenido cientifico que hayas visto en TV.}

En este apartado se refieren 311 respuestas, correspondientes a 55 títulos diferentes de los que sólo 10 son programas con contenido científico, en los 173 cuestionarios analizados. Hay un total de 28 cuestionarios sin referencias en este apartado o con respuesta explícita «no he visto».

Tanto en la pregunta 3 como en la 4 aparecen prácticamente las mismas referencias, pero cambian mucho las frecuencias. Así, el número de referencias de series de médicos e investigación policial como CSI, House es de 204, mucho mayor que en la pregunta 3, lo que indica una tendencia a clasificarlas como series con contenido científico. Asimismo, a documentales y programas o canales científicos se refieren sólo 32 referencias, un número bajísimo comparado con las referencias a series, situación que puede ser reflejo tanto del desinterés del alumnado como de la escasa presencia de programas de contenido científico en los medios españoles.

Un primer análisis de los anteriores resultados para las 4 preguntas propuestas nos lleva a concluir los siguientes puntos:

- Los alumnos, en general, conocen la CF por las películas de este género más que por la oferta de TV o la literatura.

- Por lo general, se mezcla la CF con la fantasía, no importando en este caso en qué formato se presente.

\section{VISIONES DE LA CIENCIA, LOS CIENTÍFICOS Y DE LA INFLUENCIA DE LA CIENCIA EN EL FUTURO.}

En cuanto a la visión de la ciencia, los científicos y la influencia de la ciencia en el futuro, analizamos las respuestas obtenidas en los cuestionarios realizados a los alumnos para cada una de las 3 preguntas propuestas.

\section{Escribe tres ideas sobre la ciencia que transmita la ciencia ficción.}

Se han obtenido un total de 171 respuestas habiendo encontrado $42(23,4 \%)$ cuestionarios NS/ NC. Con estos datos se calcula una media de 1,0 respuestas por cuestionario, lo que pone de manifiesto la escasa visibilidad de las ciencias para el alumnado. Si en las películas de CF, en las que están muy presentes los avances científicos y tecnológicos, no los perciben, en la vida cotidiana aún menos, se dan como algo natural.

Como ésta y las 2 siguientes son cuestiones de opinión, en el análisis de los resultados se encontró una gran diversidad de respuestas que fue necesario clasificar en categorías, utilizando en este caso unas bastante usuales en la sociología de la ciencia y muy relacionadas con las actitudes de los alumnos hacia la ciencia, que aparecen recapituladas en Solbes (1999) o en Fdez-Rañada (2003). Exponemos en la tabla 1 los resultados obtenidos. 
Tabla 1.

\begin{tabular}{|l|c|c|}
\hline $\begin{array}{l}\text { IDEAS DE LOS ALUMNOS SOBRE LA CIENCIA } \\
\text { Y LA TECNOLOGÍA EN LA CF }\end{array}$ & N.o REF. & $\begin{array}{c}\text { \% y S RESPECTO AL N.o } \\
\text { DE RESPUESTAS (171) }\end{array}$ \\
\hline IDEAS NEUTRAS & 53 & $31,0(3,2)$ \\
\hline IDEAS POSITIVAS & 42 & $24,6(2,9)$ \\
\hline IDEAS AMBIVALENTES & 29 & $17,0(2,6)$ \\
\hline OTROS & 29 & $17,0(2,6)$ \\
\hline IDEAS NEGATIVAS & 18 & $10,5(2,1)$ \\
\hline No contesta/no sabe & $42(23,4 \%$ de los 173 cuestionarios $)(2,9)$ \\
\hline
\end{tabular}

Un 24,6\% de las respuestas tienen valoraciones positivas sobre las ciencias. En ellas encontramos las ideas de que las ciencias conllevan «un mundo mejon», "mejores condiciones de vida», contienen palabras como «avance» $\mathrm{o}$ "progreso» o los alumnos aluden a mejoras concretas. Un 10,5\% de las respuestas se pueden clasificar como negativas, dado que asocian la ciencia con "catástrofes», "guerras», «destrucción» o "dominio sobre las personas".

El 31\% de las respuestas hablan de adelantos tanto en el campo científico como tecnológico, a los cuales los alumnos no han dado connotaciones positivas o negativas. Por eso consideramos que estas respuestas dan la idea de que la ciencia que transmite la CF es neutra. Y, por último, un 17\% de las respuestas se pueden clasificar como ambivalentes, es decir, incluyen las dos tendencias, positiva y negativa, a la vez, lo que posiblemente refleje en mayor medida la realidad de la ciencia y de sus interacciones con la sociedad (Solbes, 1999; Fernández-Rañada, 2003).

Aparecen algunas respuestas irreales, casi mágicas, que relacionan la ciencia con «viajes en el tiempo», "nada es imposible», "guerras entre planetas", junto a respuestas más realistas como: "mal enfocada puede causar destrucción» $\mathrm{O}$ "contaminación», puede proporcionar «soluciones a enfermedades», "queda mucho por descubrir», etc.

En las entrevistas realizadas, para profundizar en los resultados del cuestionario, se confirma la visión de avance que perciben los alumnos. Los comentarios sobre la ciencia siguen siendo favorables, pero siempre se apostilla la idea de que un uso inadecuado de la misma podría llevar a desastres. Preguntando sobre la responsabilidad de ese uso, aparecen respuestas tan variadas como la sociedad, los políticos o los científicos. Se matizan las respuestas negativas, de forma que no ven las catástrofes que suceden en las películas como posibles o las ven muy lejanas ("no tan a corto plazo como lo ponen, pero puede pasar», "podría ser real a largo plazo si no hay control») y piensan que la sociedad conseguirá sobreponerse a cualquier desastre.

\section{Escribe tres ideas sobre los cientificos que transmita la ciencia ficción.}

El número de respuestas obtenidas ha sido de 156, habiendo encontrado 44 cuestionarios NS/NC. Esto hace un total de 0,9 respuestas por cuestionario.

Como en el caso de la cuestión anterior, se ha hecho necesario clasificar las respuestas en ítems más generales y las pautas que se han encontrado son las que se detallan en la tabla 2 . 
Tabla 2 .

\begin{tabular}{|l|c|c|}
\hline $\begin{array}{l}\text { IDEAS DE LOS ALUMNOS SOBRE LOS CIENTÍFICOS } \\
\text { EN LA CF }\end{array}$ & N.o REF. & $\begin{array}{c}\text { \% y S RESPECTO AL N.o } \\
\text { DE RESPUESTAS (156) }\end{array}$ \\
\hline IDEAS FAVORABLES & 74 & $47,4(3,5)$ \\
\hline IDEAS DEFORMADAS O EXAGERADAS & 55 & $35,3(3,4)$ \\
\hline IDEAS DESFAVORABLES & 20 & $12,8(2,4)$ \\
\hline OTRAS & 7 & $4,5(1,5)$ \\
\hline No contesta & $44(25,4 \%$ de los 173 cuestionarios) $(3,2)$ \\
\hline
\end{tabular}

Un 47,4\% de las respuestas son visiones favorables sobre los científicos, indicando por ejemplo que «saben mucho», «son responsables y estudiosos», «intentan salvar personas», "bien pagados», "están muy motivados", etc.

Pero un $35,3 \%$ de las respuestas implican visiones deformadas o exageradas y un 12,8\% visiones desfavorables. Así, se menciona que los científicos son "egoistas», "gente mala», con "malos modales» (sic), "no piensan en los resultados y no se hacen responsables de ellos», etc., o que «se pasan la vida en el laboratorio", "cientifico loco", "viven en un mundo irreal», "quieren dominar el mundo", etc.

Es decir, se da un equilibrio entre ambas posturas, aunque en las películas más vistas, según el ítem 1, apenas aparecen los científicos (Star Wars, Matrix) o se da una visión negativa de los mismos en las películas de superhéroes (X-men, Spiderman, Hulk), que tanto éxito tienen hoy en día. En ellas, el antagonista suele ser un científico que enloquece y quiere dominar el mundo o que, habiendo descubierto un «arma» poderosa, la emplea para enriquecerse y acumular poder. Esta imagen de los científicos puede constituir una base para la asimilación de visiones deformadas y exageradas de la ciencia y los científicos.

Mayoritariamente las respuestas no explicitan nada sobre el género de los científicos: son respuestas impersonales, cómo "piensan», «creen», «saben», etc., o mantienen el género masculino de la pregunta. Son interesantes un $25 \%$ de respuestas que incluyen palabras como gente, personas y hombres y mujeres.

7. Escribe tres ideas sobre la capacidad de la ciencia para influir en el futuro que transmita la ciencia ficción.

El número de respuestas obtenidas en los 173 cuestionarios analizados ha sido de 277, habiendo encontrado 45 cuestionarios con esta pregunta en blanco o con la respuesta explícita: «no sé». Esto hace un total de 1,6 respuestas por cuestionario.

Detallamos en la tabla 3 los resultados obtenidos.

Tabla 3.

\begin{tabular}{|l|c|c|}
\hline $\begin{array}{l}\text { IDEAS DE LOS ALUMNOS SOBRE LAS CONSECUEN- } \\
\text { CIAS DE LA ACTUACIÓN CIENTÍFICA EN EL FUTURO }\end{array}$ & N. ${ }^{\circ}$ REF. & $\begin{array}{c}\text { \% y S RESPECTO AL N. } \\
\text { DE RESPUESTAS }(277)\end{array}$ \\
\hline IDEAS OPTIMISTAS & 170 & $61,4(2,7)$ \\
\hline IDEAS NEUTRAS & 61 & $22,0(2,3)$ \\
\hline IDEAS PESIMISTAS & 25 & $9,0(1,6)$ \\
\hline UTOPÍAS & 21 & $7,6(1,5)$ \\
\hline No contesta & $45(26 \%$ de los 173 cuestionarios $)(2,1)$ \\
\hline
\end{tabular}


Encontramos en un $61,4 \%$ de las respuestas que la idea que los alumnos tienen en función de la CF sobre las implicaciones de los avances científicos en el futuro es mayoritariamente optimista. Los alumnos han dado respuestas como: "modernidad", "la ciencia es buena», "resolución de problemas", "no dependencia del petróleo y centrales nucleares», "mejoras económicas", "supervivencia». Un $22 \%$ de las respuestas se han categorizado como neutras porque hablan de progreso, pero sin connotaciones optimistas o pesimistas: «inteligencia artificial», "la tecnología sustituye al hombre», "influye en el pensamiento», "cambio social».

En ideas pesimistas hemos incluido las repuestas que conlleven destrucción, guerra, mala utilización de recursos científicos y tecnológicos, y podemos señalar que son muy escasas ( $9 \%)$.

Por último, incluimos en la categoría utopías todas las ideas que sean científica o tecnológicamente imposibles o impliquen situaciones irreales (como «viajes en el tiempo», «inmortalidad», etc.). Constatamos que también son muy escasas $(7,6 \%)$ y contrastan con la mayoría de respuestas que hablan de un futuro plausible, como mejoras en la calidad de vida, mejoras en medicina, medioambientales, tecnológicas... aunque denoten optimismo, o pesimismo (destrucción medioambiental, guerras...).

Estos términos se confirman en las entrevistas realizadas cuando se les pregunta a los alumnos cómo ven el futuro en relación con la ciencia, a pesar de que aparecen respuestas como «destruido», o «muy tecnificado y catastrófico", son más los alumnos que lo perciben como mejor respecto al presente: "la medicina avanzará más», "la ciencia solucionará muchos problemas aunque creará otros». Cuando se les pregunta si podría ser real, la respuesta es siempre muy parecida: "no a corto plazo pero si a largo plazo».

En el cuestionario, por último, se pidió a los alumnos una valoración de 0 a 10 de la CF en general y en distintos formatos y se obtuvieron los resultados que aparecen en la tabla 4.

Un primer análisis de los resultados obtenidos con esta muestra de alumnos nos da los siguientes resultados:

- Los hombres en general están más interesados en la CF que las mujeres.

- Las personas que eligen la opción de Ciencias asignan mayor puntuación a la CF que las personas que eligen las opciones de Humanidades y Ciencias sociales.

- Los resultados anteriores se mantienen a lo largo de los cursos, variando ligeramente con la edad, ya que la puntuación es un poco más elevada en los cursos superiores.

- El interés por la CF es mayor en el caso de las películas, disminuyendo la puntuación cuando se trata de series de TV y suspendiendo, con una puntuación por debajo de 5 , para la literatura de ciencia ficción. Sólo en el caso de los alumnos de $2 .^{\circ}$ de bachillerato de ciencias, la puntuación de la literatura de CF es comparable a la puntuación de las películas y las series de TV, siendo estas últimas a las que menos nota asignan. Este resultado lo hemos de contrastar con los resultados que se detallan a continuación en los siguientes apartados. En principio, pues, se confirma la hipótesis del interés por la ciencia ficción en formato de cine, por encima de los demás formatos. 
Tabla 4.

\begin{tabular}{|c|c|c|c|c|c|c|c|}
\hline CURSO & MODALIDAD & SEXO & $\begin{array}{c}\text { N. }{ }^{\circ} \\
\text { DATOS }\end{array}$ & $\begin{array}{l}\text { INTERÉS } \\
\text { CF en } \\
\text { general }\end{array}$ & $\begin{array}{c}\text { INTERÉS } \\
\text { CINE } \\
\text { DE CF }\end{array}$ & $\begin{array}{c}\text { INTERÉS } \\
\text { SERIES TV } \\
\text { DE CF }\end{array}$ & $\begin{array}{c}\text { INTERÉS } \\
\text { LITERATURA } \\
\text { DE CF }\end{array}$ \\
\hline \multicolumn{3}{|c|}{ Resultados generales } & 173 & $6,2(3,4)$ & $6,7(3,8)$ & $5,7(3,7)$ & $4,2(4,1)$ \\
\hline \multirow{3}{*}{$3 .^{\circ} \mathrm{ESO}$} & & Todos & 55 & 5,5 & 6,5 & 5,8 & 3,8 \\
\hline & & Hombres & 35 & 6,2 & 7,1 & 6,2 & 4,3 \\
\hline & & Mujeres & 19 & 4,3 & 5,4 & 5,2 & 3,1 \\
\hline \multirow{6}{*}{$4 .^{\circ} \mathrm{ESO}$} & Todos & Todos & 38 & 5,3 & 6,1 & 5,8 & 4,0 \\
\hline & & Hombres & 17 & 5,4 & 7,4 & 6,8 & 4,8 \\
\hline & & Mujeres & 21 & 5,3 & 5,1 & 5,4 & 3,4 \\
\hline & Ciencias & Todos & 16 & 5,8 & 5,5 & 5,9 & 3,0 \\
\hline & & Hombres & 8 & 7,0 & 7,4 & 7,0 & 4,1 \\
\hline & & Mujeres & 8 & 4,5 & 3,6 & 4,7 & 1,9 \\
\hline \multirow{9}{*}{$\begin{array}{l}1 .^{\circ} \\
\text { bachillerato }\end{array}$} & Todos & Todos & 65 & 6,7 & 7,2 & 6,5 & 4,6 \\
\hline & & Hombres & 59 & 7,3 & 7,8 & 6,8 & 4,1 \\
\hline & & Mujeres & 26 & 5,9 & 6,5 & 6,0 & 5,4 \\
\hline & Ciencias & Todos & 35 & 7,4 & 7,7 & 7,1 & 4,8 \\
\hline & & Hombres & 22 & 8,1 & 8,3 & 7,5 & 4,6 \\
\hline & & Mujeres & 9 & 6,2 & 6,8 & 6,2 & 5,3 \\
\hline & Hum. y CCSS & Todos & 30 & 6,0 & 6,5 & 5,7 & 4,5 \\
\hline & & Hombres & 13 & 6,2 & 6,9 & 5,5 & 3,2 \\
\hline & & Mujeres & 17 & 5,8 & 6,3 & 5,9 & 5,4 \\
\hline \multirow{9}{*}{$\begin{array}{l}2 .^{\circ} \\
\text { bachillerato }\end{array}$} & Todos & Todos & 16 & 6,0 & 6,8 & 5,2 & 4,9 \\
\hline & & Hombres & 3 & 7,5 & 7,7 & 4,7 & 5,3 \\
\hline & & Mujeres & 12 & 5,9 & 6,6 & 5,2 & 4,9 \\
\hline & Ciencias & Todos & 4 & 7,7 & 8,0 & 6,0 & 7,3 \\
\hline & & Hombres & 2 & 7,5 & 7,5 & 5,5 & 7,0 \\
\hline & & Mujeres & 4 & 6,3 & 7,7 & 5,7 & 7,7 \\
\hline & Hum. y CCSS & Todos & 10 & 5,4 & 6,2 & 5,1 & 3,6 \\
\hline & & Hombres & 1 & Sin dato & 8,0 & 3,0 & 2,0 \\
\hline & & Mujeres & 8 & 5,6 & 6,0 & 5,1 & 3,9 \\
\hline \multirow{4}{*}{$\begin{array}{l}\text { Todos } \\
\text { los cursos }\end{array}$} & \multicolumn{3}{|l|}{ CIENCIAS } & 6,9 & 7,1 & 6,6 & 4,4 \\
\hline & \multicolumn{3}{|l|}{ Hum. y CCSS } & 5,9 & 6,5 & 5,6 & 4,2 \\
\hline & \multicolumn{3}{|l|}{ MUJERES } & 5,3 & 5,8 & 5,6 & 4,2 \\
\hline & \multicolumn{3}{|l|}{ HOMBRES } & 6,8 & 7,4 & 6,5 & 4,3 \\
\hline
\end{tabular}




\section{Resultados de profesores}

Paralelamente al estudio realizado con alumnos, se hizo un sondeo a 35 profesores en formación (CAP) y 21 en activo. Se les preguntó, sobre la CF que conocen, en formato cinematográfico, series de televisión y literario, preguntas que coinciden con las preguntas 1, 2 y 3 del cuestionario del alumnado.

Resumimos brevemente los resultados encontrados: no hay diferencias significativas entre profesores en activo y en formación en estos ítems y los profesores realizan 331 referencias a películas de CF correspondientes a 73 títulos. En concreto, siguen dominando en número de referencias las películas de la saga de La guerra de las Galaxias, junto con Regreso al Futuro, Matrix... pero películas clásicas como Metrópolis, Blade Runner, 2001: Odisea en el espacio, Yo robot..., tienen muchas más referencias.

Encontramos 38 referencias a series de CF correspondientes a 20 títulos y 67 referencias a libros de CF correspondientes a 38 títulos. Nuevamente se nota un mayor conocimiento, se citan autores antiguos como Verne (Viaje al centro de la tierra, 20.000 leguas de viaje submarino, De la Tierra a la Luna), Wells (La máquina del tiempo, La guerra de los mundos) o más literarios como Orwell (1984) y Pedrolo, aunque curiosamente no se menciona Un mundo feliz. Aparecen clásicos del género, como Dune de Herbert, La fundación de Asimov entre los más citados, junto con Jurasic Park de Crichton y Contact, escrita por el científico y divulgador Carl Sagan. Además, se mencionan algunas novelas como Neuromante de Gibson, Hyperion de Simmons o El juego de Ender de Scott Card, recientes pero ya clásicas dentro del género de la CF.

Es decir, duplican a los alumnos en referencias a libros, los superan claramente en referencias cinematográficas y sólo ligeramente en el caso de series. Por otra parte, apenas hay confusión en el profesorado entre fantasía y CF.

Una vez constatado su conocimiento de la CF, se les plantea qué podría aportar la ciencia ficción en la enseñanza de las ciencias y qué actividades se podrían realizar en caso de utilizar este recurso. Tampoco encontramos diferencias significativas entre los dos grupos respecto a estos ítems y por eso también se presentan conjuntamente.

¿Qué crees que puede aportar la ciencia ficción a la enseñanza de las ciencias?

El número total de respuestas para esta pregunta ha sido de 129 (2,3 por profesor). Los resultados han sido los que aparecen en la tabla 5.

Como vemos, un $38 \%$ de las respuestas dadas se refiere directamente a la mejora en la motivación y el interés de los alumnos por las asignaturas de ciencias. Hemos clasificado dentro de este apartado repuestas como: "despertar la curiosidad y el interés por la ciencia» de la que ha habido 39 referencias de las 49 totales en este apartado, "motivación para elegir la carrera de ciencias", "acercar la ciencia al público en general», "abrir la mente del alumno al mundo que le rodea, a indagar»...Y sin embargo, el $18,6 \%$ de las respuestas se refieren al aprendizaje en sí: "mejor comprensión de conceptos y teorias», "comprender fenómenos que no se pueden hacer en el laboratorio", "ejemplos de conceptos estudiados»....

Encontramos que profesores en activo y formación consideran la CF tanto como vehículo de aprendizaje como de motivación, ayudando con la diversificación de actividades en clase y contribuyendo a una dinámica en el aula más amena y entretenida.

Otro resultado que consideramos sintomático es que en ningún cuestionario analizado se deja esta pregunta por responder, lo que indica claramente que los profesores admiten que la utilización de la ciencia ficción como recurso didáctico es válida y útil. 
Tabla 5 .

\begin{tabular}{|l|c|c|}
\hline APORTE DE LA CF A LA ENSEÑANZA & N.o DE RESULTADOS & $\begin{array}{c}\text { \% y S RESPECTO } \\
\text { AL N. }{ }^{\circ} \text { DE RESPUESTAS (129) }\end{array}$ \\
\hline Motivación e interés. & 49 & $38,0(4,3)$ \\
\hline Aprendizaje. & 24 & $18,6(3,4)$ \\
\hline Relaciones CTSA y futuro. & 23 & $17,8(3,4)$ \\
\hline Visión de la ciencia y los científicos. & 20 & $15,5(3,2)$ \\
\hline Relación ciencia-ciencia ficción & 11 & $8,6(2,5)$ \\
\hline Otros. & 2 & $1,6(1,1)$ \\
\hline
\end{tabular}

Propón actividades que utilicen la ciencia ficción para enseñar ciencias.

Cuando se les pregunta por actividades concretas, el número total de respuestas disminuye a 90 (1,6 por profesor), lo que puede ser un indicador de que no conocen ni hacen (los que están en activo) muchas.

Los resultados clasificados por categorías y los porcentajes correspondientes se indican en la tabla 6 .

Vemos que un $37,8 \%$ de las respuestas se dirige a la relación de la ciencia ficción con la ciencia: "debates para determinar lo que es ciencia en la $C F$ ", "analizar pasajes para determinar la veracidad de la CF", "plantear preguntas y problemas sobre ciencia y/o futuro", considerando que el cine de ciencia ficción puede servir para proponer actividades de debate, análisis... sobre los que apoyar esta relación. Incluso se ha propuesto "escribir un relato de $C F$ », que fue una propuesta didáctica realizada en los años 50 en la antigua URSS para fomentar la elección de las carreras de ciencias y que, como ya hemos comentado anteriormente en el marco teórico, diversos investigadores han utilizado.

Un 26,7\% de las respuestas propone también actividades de aprendizaje y resolución de problemas, dirigidas por tanto a la asimilación de conceptos y apoyo de las clases convencionales, integrando esas actividades en la dinámica habitual, o como resumen o aplicación de los conceptos ya estudiados: «extraer fenómenos físicos de peliculas y proponerlos como introducción al tema por parte del profesor o del alumno", "explicación de situaciones», "resolución de problemas»...

Queremos destacar asimismo las pocas respuestas que propongan actividades dirigidas en el sentido de detección de errores, siendo una de las causas que más investigación ha generado en torno a la relación de la ciencia y la ciencia ficción.

Tabla 6.

\begin{tabular}{|l|c|c|}
\hline ACTIVIDADES PROPUESTAS & N. ${ }^{\circ}$ DE RESULTADOS & $\begin{array}{c}\text { \% y S RESPECTO } \\
\text { AL N. }{ }^{\circ} \text { DE RESPUESTAS (90) }\end{array}$ \\
\hline Relación ciencia-ciencia ficción. & 34 & $37,8(4,9)$ \\
\hline Aprendizaje conceptual y de resolución de problemas. & 24 & $26,7(4,4)$ \\
\hline Problemas tecnológicos y técnicos. & 10 & $11,1(3,1)$ \\
\hline Otros. & 7 & $7,8(2,7)$ \\
\hline Detección de errores. & 5 & $5,6(2,3)$ \\
\hline Actualidad. & 5 & $5,6(2,3)$ \\
\hline No sabe / no contesta. & 5 & $5,6(2,3)$ \\
\hline
\end{tabular}




\section{Análisis de textos}

De los 31 libros de secundaria y bachillerato analizados, en 22 de ellos no hemos encontrado ni una sola referencia a la CF, ni en fotografías, comentarios o textos, actividades o referencias a webs, ni en el libro de texto del alumno, ni en el libro del profesor o en cuadernos de actividades o CD-ROM en el caso de haberlos. En 5 libros (3 de física y química, 1 de tecnología y 1 de biología y geología) hemos encontrado 1 elemento de CF (foto, texto o cuestión-problema). En 3 libros (1 de tecnología y 2 de física y química) se evidencian 2 elementos y sólo en 1 libro (física y química) se han encontrado 3 elementos de CF.

Entre estos elementos destacamos una foto de Superman localizada en un texto complementario sobre el descubrimiento del mineral jadarita, cuya fórmula química es muy similar a la fórmula del mineral ficticio kryptonita. Asimismo, una foto de la nave Enterprise acompañando a un texto complementario sobre las fuentes de energía de las naves, ambas en un libro de física y química de $10^{\circ}$ de bachillerato publicado en 2008. En dicho libro se ha encontrado un problema sobre la distancia que podía recorrer el capitán Nemo en su viaje submarino. Otro problema encontrado hace referencia a las rpm (revoluciones por minuto) que debería dar la estación espacial de 2001, Odisea en el espacio para simular la gravedad terrestre.

En los libros de tecnología se ha encontrado una actividad que consiste en el diseño de un coche del año 2050, un texto que hace referencia a las leyes de la robótica de Isaac Asimov y otro texto que menciona un ciclo de cine de ciencia ficción con ejemplos de películas como Matrix y Blade Runner.

Dado que los libros de texto contribuyen en gran medida a establecer los contenidos que se enseñan, esto nos indica, junto al escaso número de actividades propuestas por el profesorado, que la CF está poco presente en las aulas, a pesar de la opinión del profesorado favorable a las mismas.

\section{CONCLUSIONES Y PERSPECTIVAS}

A continuación, se exponen las conclusiones que se extraen a la vista de los resultados.

- En cuanto a las preguntas ¿qué entienden los y las estudiantes por ciencia ficción? y ¿qué ciencia ficción conocen?, podemos decir que el alumnado analizado está más familiarizado con el cine de ciencia ficción (3,3 referencias por cuestionario) que con las series de TV de CF (1) o con la literatura de este género $(0,6)$. Muchos alumnos confunden CF con fantasía-magia, llegando a un $51 \%$ de referencias de magia en el caso de las novelas.

- Encontramos en promedio, y teniendo en cuenta que se han analizado un gran número de cuestionarios pertenecientes a una amplia gama de edades, niveles y procedencias de alumnos, bastante interés por la CF (una media de 6,2), superada en el caso del cine de CF (6,7), menor en el caso de las series $(5,7)$ y desinterés en el caso de las novelas $(4,2)$. Esta preferencia por el cine y desinterés por la lectura coincide con las encuestas realizadas por el Ministerio de Cultura, antes mencionado.

- Respecto a la imagen de la ciencia y de los científicos y qué visión sobre el futuro captan los alumnos a través de la CF, hay muy pocas respuestas sobre la imagen de la ciencia que transmite la CF (1,0 respuesta por cuestionario cuando se pedían 3) lo que nos habla de la escasa visibilidad de las ciencias para los alumnos encuestados, a pesar de su gran presencia en las obras de CF.

- También hay pocas respuestas sobre la imagen que tiene el alumnado de los científicos a través de la CF (0,9 respuestas por cuestionario) y casi la mitad de las ideas sobre los científicos que 
aparecen son exageradas o desfavorables, lo que puede poner de manifiesto la influencia del científico perverso, loco o instrumento ciego del poder característico de la CF.

- No ocurre lo mismo con la idea de la influencia que la ciencia puede tener en el futuro transmitida por el género de CF (1,6 respuestas por cuestionario), y en contra de la imagen catastrofista del futuro que se presenta en gran parte de las películas de CF, los alumnos no opinan que el futuro vaya a ser peor que el presente debido a la intervención científica.

- Los profesores, en formación y en ejercicio analizados, opinan sobre la utilización de la ciencia ficción como recurso didáctico que las actividades relacionadas con el cine de CF serían útiles a la hora de mejorar la actitud de los alumnos en las clases de ciencias, y también, aunque en menor grado, para mejorar el aprendizaje de conceptos y resolución de problemas.

- Y, por último, en cuanto a la utilización de la ciencia ficción en los libros de texto analizados, hemos encontrado que, a pesar de la opinión favorable del profesorado, en los textos apenas hay ejemplos de utilización de CF para realización de actividades como cuestiones, problemas, comentarios de texto o visionado de películas.

Queda pues pendiente, para un trabajo posterior, un análisis exhaustivo de alguna de las películas más vistas por los alumnos, en la línea de Perales y Vílchez (2005), con el cual podremos confirmar si dichas películas transmiten o no visiones deformadas de ciencia y científicos y visiones catastrofistas del futuro.

También el estudio por sexos del conocimiento de la CF de los profesores, estudio que ampliaría los resultados obtenidos en este trabajo. Proponemos seguir analizando libros y publicaciones relacionadas directamente con la Educación Secundaria Obligatoria y el bachillerato, así como publicaciones realizadas en el campo de la didáctica de las ciencias experimentales en relación con la utilización del cine de CF en la enseñanza.

Propondríamos actividades de aprendizaje basadas en películas o series y no en libros de CF, por ser las que más interesan y, en consecuencia, las que más influyen. Por último, queremos averiguar si dichas actividades conllevarían una mejora en la actitud y en el aprendizaje de los alumnos, por lo que nos proponemos la realización de actividades de este tipo y el estudio de mejoras en la actitud, la participación en clase y el aprendizaje de los conceptos realizando un seguimiento de los alumnos a lo largo de varios cursos. 


\section{REFERENCIAS BIBLIOGRÁFICAS}

BACAS, P., MARTÍN, M. J., PERERA, F. y PIZARRO, A. M. (1997). Una propueta didáctica para bachillerato: Física y Ciencia Ficción. Revista española de física, 11(4), pp. 31-37.

BACAS, P., MARTÍN M. J., PERERA F. y PIZARRO A. M. (1993). Física y ciencia ficción. Madrid: Akal. BANET, E. (2007). Finalidades de la educación científica en secundaria: opinión del profesorado sobre la situación actual. Enseñanza de las Ciencias, 25(1), pp. 5-20.

BARCELO, M. (2000). Paradojas: ciencia en la ciencia ficción. Madrid: Equipo Sirius.

- (2005). Paradojas: ciencia en la ciencia ficción II. Madrid: Equipo Sirius.

CLUTE, J. y NICHOLLS, P. (1995). The Encyclopedia of Science Fiction. Londres: Orbit.

DUNBAR, R. (1999). El miedo a la ciencia. Madrid: Alianza Editorial.

ELIAS, C (2008). La ciencia estrangulada. Barcelona: Debate.

FENSHAM, P. J. (2004). Beyond Knowledge: Other Scientific Qualities as Outcomes for School Science Education, en Janiuk, R. M. y Samonek-Miciuk, E. (ed. ). Science and Technology Education for a Diverse World - dilemmas, needs and partnerships. International Organization for Science and Technology Education (IOSTE) XIth Symposium Proceedings, pp. 23-25. Lublin, Polland: Maria Curie-Sklodowska University Press.

FERNÁNDEZ-RAÑADA, A. (2003). Los muchos rostros de la ciencia. México: Fondo de Cultura Económica.

FURIÓ, C. y CARNICER, J. (2002). El desarrollo profesional del profesor de Ciencias mediante tutorías de grupos cooperativos. Estudio de ocho casos. Enseñanza de las Ciencias, 20(1), pp. 47-73.

FURIÓ, C., SOLBES, J. y FURIÓ, C. (2008). Towards of effective ongoing training programmes for science teachers. Problems of educations in the 21st Century, 6, pp. 60-71.

FURIÓ, C. y VILCHES, A. (1997). Las actitudes de los estudiantes hacia las ciencias y las relaciones CTS, en Del Carmen (coord. ). La enseñanza y el aprendizaje de las ciencias de la naturaleza en la educación secundaria. Barcelona: Horsori, pp. 47-71.

GARCÍA, F. J. (2006). Cuando los mundos chocan. Revista Eureka sobre Enseñanza y Divulgación de las Ciencias, 3(2), pp. 268-286.

HELlSTRAND, A. y OTT, A. (1995). The utilization of fiction when teaching the theory of relativity. Physics Education, 30(5), pp. 284-286.

HEWSON, P. W. y HEWSON, M. G. (1987). Science teachers' conceptions of teaching: implications for teachers education, International Journal of Science Education, 9(4), pp. 425-440.

JOSÉ, J. (2006). Científics a 24 fotogrames per segon. Mètode, 48, pp. 77-82.

KAKALIOS, J. (2006). La física de los superhéroes. Barcelona: Ediciones Robinbook.

MATTHEWS, M. R. (1990). History, Philosophy, and Science Teaching: A rapprochement. Studies in Science Education, 18, pp. 25-51.

MOReno, M. (2006). El cinema i la ciencia: crònica d'un desamor. Mètode, 48, pp. 58-64.

MORENO, M. y JOSÉ, J. (2002). De King Kong a Einstein: la física en la ciencia ficción. Barcelona: Servicio de Publicaciones UPC.

NICHOLLS, P. (1987). La ciencia en la ciencia ficción. Barcelona: Orbis.

PALACIOS, S. L. (2007). El cine y la literatura de ciencia ficción como herramientas didácticas en la enseñanza de la física: una experiencia en el aula. Revista Eureka sobre Enseñanza y Divulgación de las Ciencias, 4(1), pp. 106-122.

PERALES, F. J. y VÍLCHEZ, J. M. (2005). The teaching of physics and cartoons: Can they be interrelated in secondary school? International Journal of Science Education, 27(14), pp. 1647-1670.

PETIT, M. F. (2008). La ciencia ficción en la enseñanza de la física y la química. Trabajo de investigación de tercer ciclo. Universitat de València. 
PORLÁN, R., RIVERO, A y MARTíN, R. (1998). Conocimiento profesional y epistemología de los profesores II: estudios empíricos y conclusiones. Enseñanza de las Ciencias, 16(2), pp. 271-289.

POZO, J. I. y GÓMEZ CRESPO, M. A. (1998). Aprender y enseñar ciencia, Morata, Madrid. PRO, A. y EZQUERRA, A. (2005). ¿Qué ciencia ve nuestra sociedad? Alambique, 43, pp. 37-49.

ROCARD, M. et al. (2007). Science education Now: A renewed Pedagogy for the future of Europe. European Communities: Belgium <http://ec. europa. eu/research/science-society/document_library/pdf_06/ report-rocard-on-science-education_en.pdf (12 de octubre de 2010)>.

SIERRA, C. E. (2007). Fortalezas epistemológicas y axiológicas de la ciencia ficción: un Potosí pedagógico mal aprovechado en la enseñanza y divulgación de las ciencias. Revista Eureka para la Enseñanza y Divulgación de las Ciencias, 4(1), pp. 87-105.

SIMPSON, R. D., KOBALA, T. R., OLIVER, J. S. y CRAWLEY, F. E. (1994). Research on the affective dimension of science learning, en Gabel, D. L. (ed. ). Handbook of Research on Science Teaching and Learning. Nueva York: McMillan Pub Co.

SJØBERG, S. y SCHREINER, C. (2006). How do students perceive science and technology? Science in School, 1, pp. 66-69.

SOLBES, J. (1999). Los valores en la enseñanza de las ciencias. Alambique, 22, pp. 97-109.

SOLBES, J., MONSERRAT, R. y FURIÓ, C. (2007). El desinterés del alumnado hacia el aprendizaje de la ciencia: implicaciones en su enseñanza. Didáctica de las Ciencias Experimentales y Sociales, 21, pp. 91-117.

SOlBES, J., SOUTO, X., TRAVER, M., JARdón, P. y RAMíreZ, S. (2004). Visión del alumnado de las TIC y sus implicaciones sociales. Investigación en la Escuela, 54, pp. 81-93.

SOLBES, J. y TRAVER, M. (2001). Resultados obtenidos introduciendo la historia de la ciencia en las clases de física y química: mejora de la imagen de la ciencia y desarrollo de actitudes positivas, Enseñanza de las Ciencias, 19(1), pp. 151-162.

SOLBES, J. y VILCHES, A. (1997). STS interactions and the teaching of physics and chemistry. Science Education, 81(4), pp. 377-386.

VÁZQUEZ, A. y MANASSERO, Ma . A. (2008). El declive de las actitudes hacia la ciencia de los estudiantes: Un indicador inquietante para la educación científica. Revista Eureka sobre Enseñanza y Divulgación de las Ciencias, 5(3), pp. 274-292.

YAGER, R. E. y PENICK, J. E. (1986). Perception of four age groups towards science classes, teachers and values of science, Science Education, 70, pp. 353-356. 


\title{
SCIENCE FICTION AND THE TEACHING OF SCIENCE
}

\author{
$M^{a}$ Francisca Petit Pérez \\ mfranpe@alumni.uv.es \\ Jordi Solbes Matarredona \\ Departament de didàctica de les Ciencies Experimentals i Socials. Universitat de València \\ jordi.solbes@uv.es
}

One of the biggest problems that we have tackled in the field of Science Didactics has been, and still is, that in society, and students especially, have a very negative image of Science, since they consider it very boring or only able for genius... This situation leads to a lack of interest for science studies.

This increasing lack of interest for Science and the poor motivation of students force teachers to search resources that, when used in the classroom, induce an attitudinal change among the students.

The mass media, TV and cinema in particular, but also literature, spread scientific content both fictitious and real, through different ways such as documentaries, series and films, comics and novels. That's why students, who are so aware of this media, «learn» through them and this way of learning reverts in the classroom.

The decision of using science-fiction cinema as a learning tool is based on the results of the survey, which depicts that young people would rather watch films and TV series than read science-fiction books.

Although some experiences exist in the use of science-fiction as a learning tool, most of them are taken into account at a university-level education and are carried out among students who have already chosen Science, so their attitude towards Science doesn't count as fact of study.

In order to carry out this preliminary study, different surveys were proposed with different kind of questions, such as attitude, assessment of science-fiction by students and high school teachers.

On the one hand, the students were asked about what science-fiction films they knew and which ones they preferred. Furthermore, they were asked to value science-fiction literature, films and TV series. On the other hand, the students were asked to suggest the vision of science, scientists and the influence of science in the future that they perceive in the science-fiction cinema.

At the same time, in this study the teachers were asked about the use of some activities based on sciencefiction films in the classroom.

Finally, a previous study on the textbooks often used by teachers in the classroom was carried out in order to determine if they contain any reference to science-fiction.

So this study is suitable as a starting point to develop learning activities in the classroom based on the sciencefiction cinema that motivates the students and, of course, help them to learn scientific concepts with a different methodology, that is, more enjoyable, pleasant and designed from their preferences. 